\title{
A modular laboratory curriculum for teaching integrated photonics to students with diverse backgrounds
}

Samuel Serna, Nancy Hidalgo, Janice Tjan, Kevin McComber, Lionel Kimerling, et al.

Samuel Serna, Nancy Hidalgo, Janice Tjan, Kevin McComber, Lionel Kimerling, Erik Verlage, Julie Diop, Juejun Hu, Sajan Saini, Anu Agarwal, Gerald Gagnon, Stefan Preble, Gregory Howland, Matthew van Niekerk, Jeff Steidle, Karl Mcnulty, Jaime Cardenas, Meiting Song, Miloš Popović, Anatoly Khilo, Pradnya Nagarkar, Farhad Vazehgoo, Ira Moskowitz, Guiru Gu, Cheryl Schnitzer, Edward Deveney, Thomas Kling, Douglas Petkie, Jacob Longacre, "A modular laboratory curriculum for teaching integrated photonics to students with diverse backgrounds," Proc. SPIE 11143, Fifteenth Conference on

Education and Training in Optics and Photonics: ETOP 2019, 111431C (2 July 2019); doi: 10.1117/12.2523867

Event: Fifteenth Conference on Education and Training in Optics and Photonics: ETOP 2019, 2019, Quebec City, Quebec, Canada 


\section{A modular laboratory curriculum for teaching integrated photonics to students with diverse backgrounds}

Samuel Serna, Nancy Hidalgo, Janice Tjan, Kevin McComber, Lionel Kimerling, Erik Verlage, Julie Diop, Juejun Hu, Sajan Saini and Anu Agarwal

AIM Photonics Academy, Department of Materials Science and Engineering and Materials Research Labs, Massachusetts Institute of Technology, Cambridge, Massachusetts 02139, USA

Gerald Gagnon

Lincoln Laboratory, Massachusetts Institute of Technology, Lexington, Massachusetts 02421, USA

Stefan Preble, Gregory Howland, Matthew van Niekerk, Jeff Steidle and Karl Mcnulty Microsystems Engineering, Rochester Institute of Technology, Rochester, New York 14623, USA

Jaime Cardenas and Meiting Song

The Institute of Optics, University of Rochester, Rochester, New York 14627, USA

Miloš Popović and Anatoly Khilo

Department of Electrical and Computer Engineering, Boston University, Boston, Massachusetts 02215, USA

Pradnya Nagarkar

Novel Materials and Coatings (NMC), Weston, Massachusetts, 02493, USA

Farhad Vazehgoo and Ira Moskowitz

Masstech Collaborative, Westborough, Massachusetts Massachusetts 01581, USA

Guiru Gu and Cheryl Schnitzer

Departments of Physics and Chemistry, Stonehill College, Easton, Massachusetts 02357, USA

Edward Deveney and Thomas Kling

Department of Physics, Bridgewater State University, Massachusetts 02325, USA

Douglas Petkie

Department of Physics, Worcester Polytechnic Institute, Worcester, Massachusetts 01609-2280, USA

Jacob Longacre

Quinsigamond Community College, Worcester, Massachusetts 01606-2092, USA

\footnotetext{
ABSTRACT

A modular laboratory curriculum with exercises for students and lesson plans for teachers is presented. Fundamentals of basic integrated photonic (IP) devices can be taught, first as a lecture-in-the-lab followed by "hands-on” laboratory

Fifteenth Conference on Education and Training in Optics and Photonics: ETOP 2019, edited by Anne-Sophie Poulin-Girard, Joseph A. Shaw, Proc. of SPIE Vol. 11143, 111431C · (C) 2019

SPIE, ICO, IEEE, OSA · CCC code: 0277-786X/19/\$18 · doi: 10.1117/12.2523867 
measurements. This comprehensive curriculum utilizes data collected from the "AIM Photonics Institute PIC education chip” that was designed specifically for the purpose of education, and was fabricated at AIM SUNY Poly. Training using this modular curriculum will be performed through the AIM Photonics Academy network in New York (NY) and Massachusetts (MA), either as a full semester course or as a condensed boot-camp. A synergistic development and delivery of this curriculum will coherently leverage multiple resources across the network and can serve as a model for education and workforce development in other Manufacturing USA institutes, as well as for overseas partners.

Keywords: Photonics, education and training, optics education, modular, curriculum, hands-on training, workforce development.

\section{INTRODUCTION}

A proliferation of new technologies in low-power cloud computing, ultra-high-speed wireless, smart sensing, and augmented imaging have begun to leverage the synergy of photonic and electronic devices working in tandem within an integrated circuit package [1,2]. As a result, critical curricular gaps are now becoming apparent in the training of engineer and technician level students for these emerging industries.

In the microelectronics industry, there has been a historic divide between circuit design and its packaging and testing, corresponding to a division between specialized labor - that of design engineers versus test and packaging technicians. However, with photonic integrated circuits (PICs), the difference between these two groups has narrowed because of similar challenges that arise from manufacturing variations of photonic circuit components and the impact of heat management within PIC chips. As shown in Fig. 1, a "hands-on" photonics curriculum is required for training both technicians and engineers to become independent thinkers who can meet practical challenges.

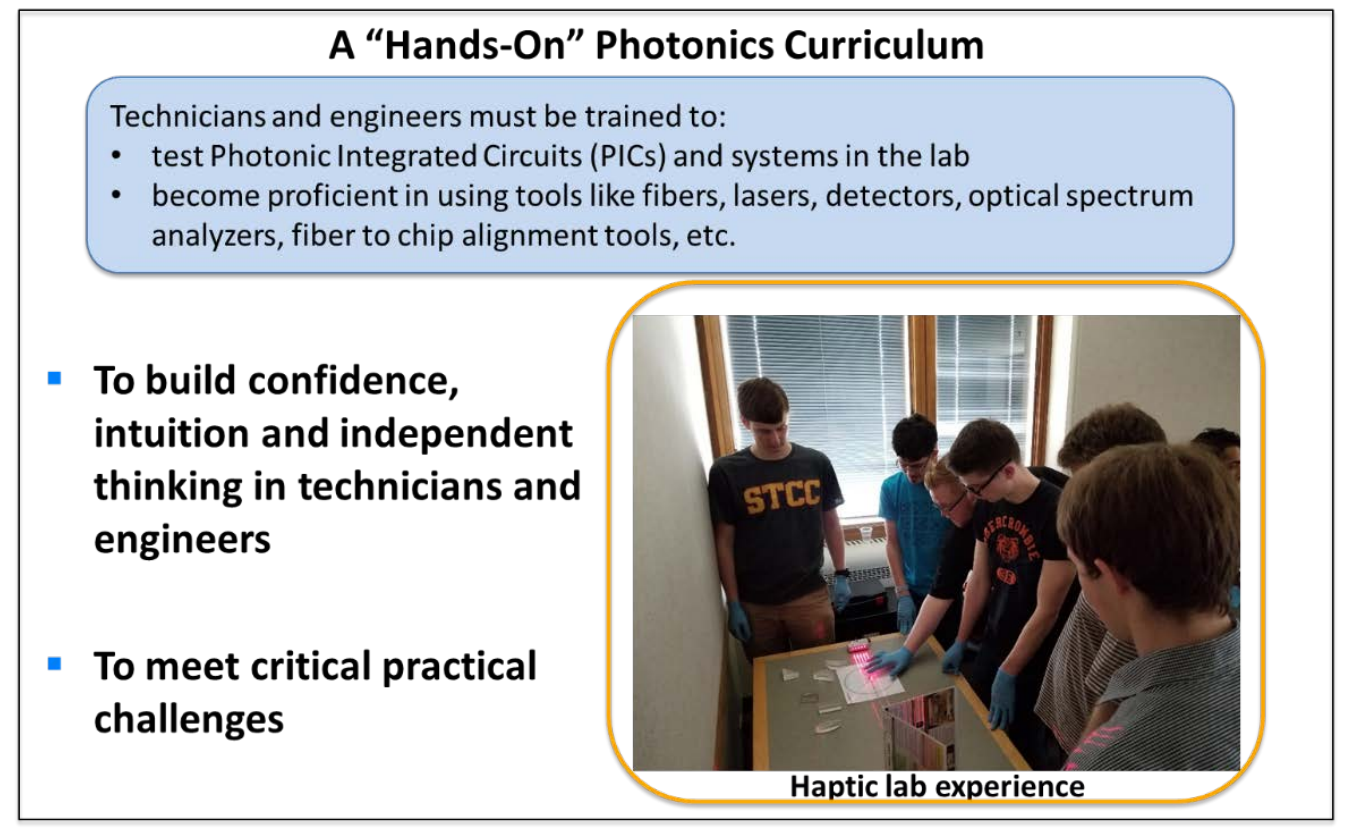

Figure 1. Technicians and engineers who have experience with hands-on characterization of Photonic Integrated Circuits (PICs) and associated tools are desired as employees in optics and photonics industries.

Worldwide, engineers training for careers in the electronics and photonics industries are thoroughly prepared in electromagnetism, advanced device and circuit design, and semiconductor technology, including fabrication and testing. Technicians are also trained in using and maintaining semiconductor fabrication and testing tools. However, the rapidly 
developing insights from the leading edge of new technologies about requirements for chip test, assembly, and packaging (TAP) - for monitoring process variation, reliability, and heat dissipation, are not adequately covered for either group.

Moreover, comprehensive laboratory classes that teach integrated photonics to a diverse audience including students from community colleges, two-year and four-year colleges, and beyond, do not exist, although the demand for a trained workforce serving the entire supply chain is rapidly increasing. This demand is fueled by (i) federally-funded AIM Photonics Institute in NY, which consists of the SUNY Poly NanoTech complex in Albany, Test Assembly and Packaging (TAP) center in Rochester, and AIM Photonics Academy at MIT; (ii) New York State investment in workforce development testing facilities at Rochester Institute of Technology (RIT) and University of Rochester (UR); and (iii) Commonwealth of Massachusetts' investment in a network of laboratories, each called a "Lab for Education and Application Prototypes” (LEAP), building on the state's manufacturing training program.

From an initial survey conducted within Massachusetts across companies and government laboratories, there is a scarcity of qualified technicians in advanced manufacturing, specifically in IP. Sufficient material for IP teaching is also lacking. Currently, no standard IP curriculum exists for instructors, and existing centralized IP materials fall short of what is needed. To address this need, we plan to make our curriculum available in modular form for technicians and engineers; both cohorts will greatly benefit from having a common foundation in IP. This content will also be designed to be comprehensive and adaptable to a wide range of instructors' backgrounds and resources. The final goal is to train technicians and/or engineers in the field of integrated photonics, in order to enable them to be effective in their workplaces.

The development of an integrated photonics curriculum is presented in Section 2. Instead of courses, the curriculum comprises five laboratory manuals, one each for a modular experiment that teaches "hands-on" skills for testing and analyzing a specific PIC device. The manuals are available either as hard copy books or through an online link. Section 2.1 of this paper identifies the basic PIC building blocks which have been chosen as teaching tools, and also includes the reasoning for such a selection. Section 2.2 discusses pedagogy, which has benefited greatly from student feedback. Section 3 presents the approach towards teaching specific concepts required for hands-on training and introduces different steps to characterize a PIC. Section 4 presents methods for assessing learning outcomes in students. Finally, Section 5 discusses plans for curriculum adoption, and the conclusions are presented in Section 6.

\section{OUTLINE OF THE CURRICULUM: TEACHING AND TRAINING A WORKFORCE FOR AN EXPANDING FIELD OF SCIENCE AND TECHNOLOGY}

Feedback about workforce training needs from the optics/integrated photonics industry was obtained from several sources, including:

(i) 2018 interviews and discussions with our industry partners,

(ii) a study performed in 2017 by RIT (https://aimphotonics.academy/workforce/workforce-needs-assessment), and

(iii) a survey conducted in 2019 by a team led by AIM Photonics Academy through a MEEP program

Summarizing from these sources, a PIC technician and/or lab engineer must be proficient in the following basic skills:

- Oral and written communication skills

- Good familiarity with Microsoft Office tools

- Gathering and organizing data using Microsoft Excel spreadsheets

- Data interpretation and presentation using Microsoft PowerPoint slides and

- $\quad$ Report-writing using Microsoft Word

- Hands-on work experience

- Lab organizational skills

- Good understanding of Environmental Health and Safety (EHS) in a lab setting

- Working/collaborating with a diverse team

- Photonics/optics-based content expertise including knowledge about

- Lenses

- Total Internal Reflection (TIR)

- Numerical Aperture (NA)

- Optical alignment on a free-space optical bench

- Single mode and multi-mode optical fibers and their use

- On-chip single-mode and multi-mode waveguides

- Sources of loss in an optical/photonic circuit (absorption, scattering coupling, bending etc.) 
- Use of components used in device characterization (lasers, Erbium-doped fiber amplifier (EDFA), visible and infrared cameras, optical spectrum analyzers (OSA's) and photodetectors)

- Characterization of an on-chip photonic device such as a waveguide, loss spiral, ring resonator and a modulator

- Guides and tips for alignment and imaging of waveguide modes

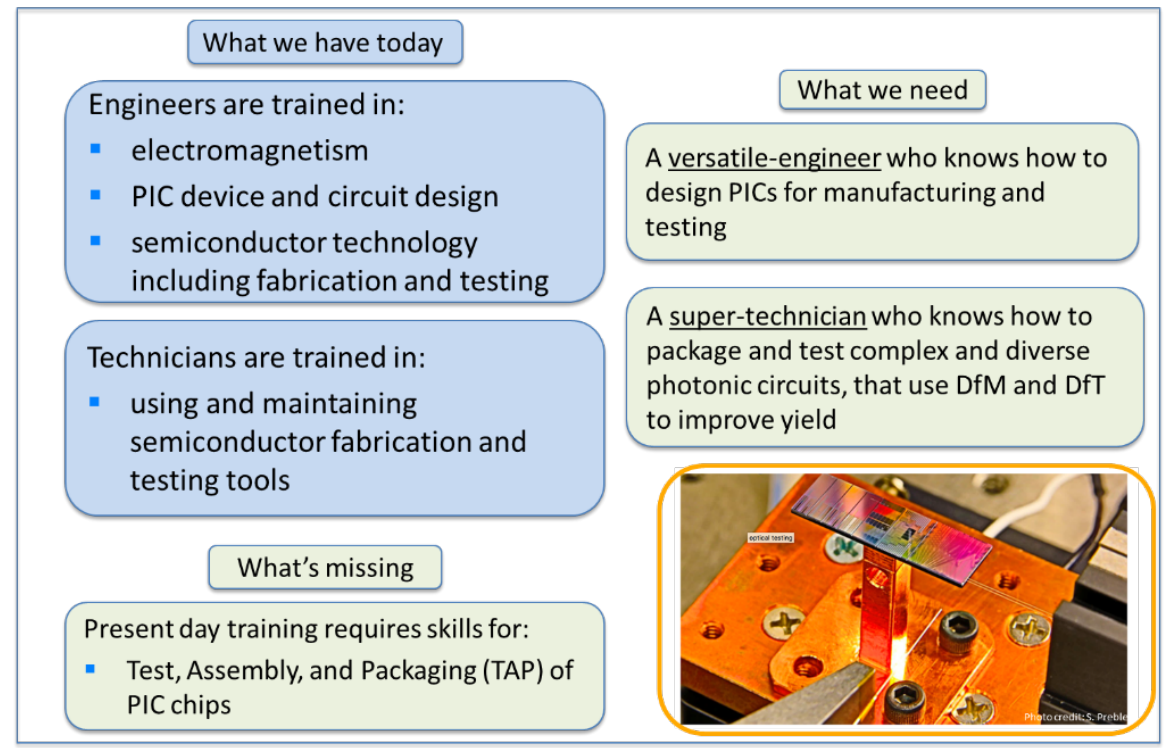

Figure 2. Identification of what is missing in photonics training today (photographic image courtesy RIT), informed the creation of the "hands-on" laboratory curriculum, where DfM and DfT stand for Design for Manufacturability and Testability, respectively.

As depicted in Fig 2, success in this emerging field of PICs will require workers to obtain a new skill-set leading either to: (i) a versatile engineer who has learned how to use design for manufacturability and testability, and/or (ii) a supertechnician who has learned how to test and analyze complex and diverse photonic circuits that use Design for Manufacturability (DfM) and Design for Testability (DfT) to improve yield.

To enable such training, a hands-on curriculum was designed and developed and comprises five laboratory manuals, one for each selected PIC device. As the field of IP grows, the need for standardized terminology and measurements becomes even more important. To address this need, the curriculum also includes a library of common words and phrases used in IP. These include information about typical defects, such as dust particles, scratches, discontinuity in waveguides among others, and a glossary of terms that are currently non-standard within the photonics community. For instance, waveguide propagation loss is reported in literature both in units of decibels per centimeter $(\mathrm{dB} / \mathrm{cm})$ and $1 / \mathrm{cm}$. The origin and need for these different units is explained in detail. When technicians and engineers who work together in an industry share a common vocabulary, important ideas such as design for manufacturability (DfM) and design for testability (DfT) can become a common goal, and lead to improved product performance and increased yields.

The comprehensive curriculum begins with a brief history of the genesis of PICs. In keeping with the needs-assessment survey results, laboratory safety training is presented before any work has started, since this is a crucial first step in handson training. Specifically, for this field, laser safety training is mandatory in all PIC characterization facilities, with every individual facility needing to follow their own safety protocols prior to laser use. Single-mode and multi-mode fibers and information about how to use and care for fibers is a prerequisite for every lab-bench characterization test using fiber technology. The remaining laboratory manuals teach how to make basic measurements to characterize PICs, and the following sections highlight examples of the pedagogy employed.

\subsection{Basic building blocks used to teach concepts in Photonic Integrated Circuits (PICs)}

Personal safety and equipment safety are emphasized at the beginning of the first laboratory manual. The standard building blocks used in this pedagogy include: (a) a straight waveguide emphasizing the coupling procedure, (b) loss 
characterization by using spirals, (c) a ring resonator, (d) a Mach-Zehnder modulator (which includes a directional coupler), and (e) a photodetector. Components such as lasers, cameras, fibers, optical spectrum analyzers and detectors are also a part of the overall photonic test circuit. Microscope images of some PIC structures from the widely available literature are shown in Fig. 3. The devices used in this work are fabricated at the SUNY Poly, Albany, NY, using a standard Silicon-on-Insulator (SOI) photonics platform. To help build the laboratory manuals, which contain student lab exercises and instructor lesson plans, measurements are made on an AIM Photonics Institute PIC education chip that contains a collection of devices (a)-(e). The laboratory exercises and lesson plan also include the modeled theoretical data as a comparative reference.

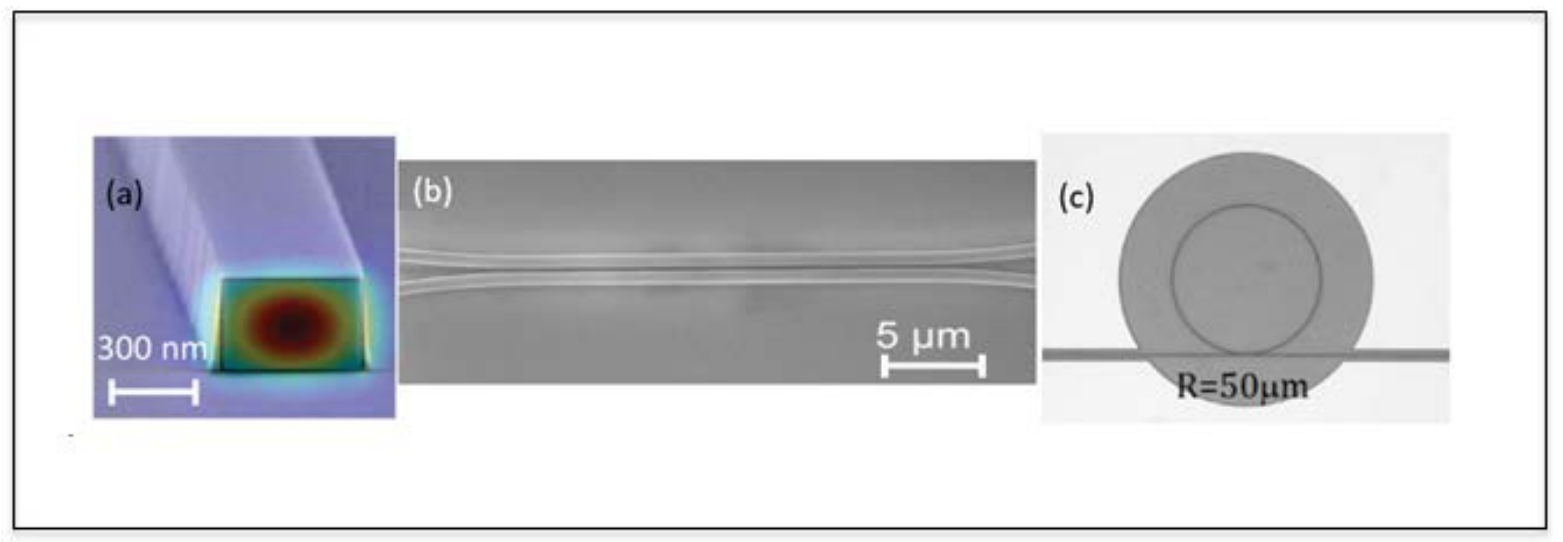

Figure 3. Examples of fabricated PIC devices: (a) Strip waveguide with the confined fundamental TE mode [3]; (b) Directional coupler; and (c) Ring resonator with $50 \mu \mathrm{m}$ radius [4].

These building blocks increase student awareness of basic PIC principles and functionalities which enable diverse applications. The laboratory manuals frequently draw analogies between the more familiar electronics and the less familiar photonics devices. For instance, students are taught that a strip waveguide on a photonic chip is used to transport photons from one part of a chip to another and is similar to a metallic wire that transports electrons across an electronic chip. Differences between the electronic and the photonic worlds are used to explain the advantages and challenges of using electromagnetic fields (photons) instead of using electrons to transmit signals. Following the same example, the particular restriction of a minimum radial bend is used to avoid losses in photonics which is not an issue in microelectronics where 90 degrees turns are possible.

Coupling a fiber to a chip is a crucial skill that is required in all PIC characterizations [5]. Both edge-coupling as well as grating coupling are presented. In fact, in recognition of the importance of coupling, an entire laboratory manual is devoted to its discussion.

As an example of the structure of each laboratory manual, an excerpt from experiment 1 (laboratory manual 1) is shown in Fig. 4. The title identifies the target audience as engineers. The (MIT) tools which are used to demonstrate the edgecoupling technique as well as the key learning objectives in the lab, are stated at the beginning. A labelled schematic of the testing system is shown in Fig. 4.1, and a photograph of the same testing system is shown in Fig. 4.2. A close-up of the input and output fibers coupled to the chip is shown in Fig. 4.3. 


\section{Lab 1: Edge-coupling Light Intoa Chip (forEngineers)}

Tools used:

- LUNA OVA 5000 Optical

Vector Analyzer

- Lens Tip Fiber

- Newport bare fiber holders

- Mounted overheadvisible

camera

- Newport stage with

three-axis alignment

- NIR Camera

- Objective

\section{Key Learning Objectives:}

In this lab, we will study the optical coupling between a lensed silica fiber and an integrated Silicon-on-Insulator (SOI) waveguide. This is called butt-coupling or end-fire coupling. This method provides broadband and polarization insensitive optical coupling. Tasks that will be covered during the lab include:

Learning parts of testing setup to couple light into a photonic chip Measuring power exiting the chip

Optimizing power coupling by adjusting fiber alignment

Learning sensitivity of coupling to misalignment

Figure 4. Excerpt of the introduction to an experiment manual.

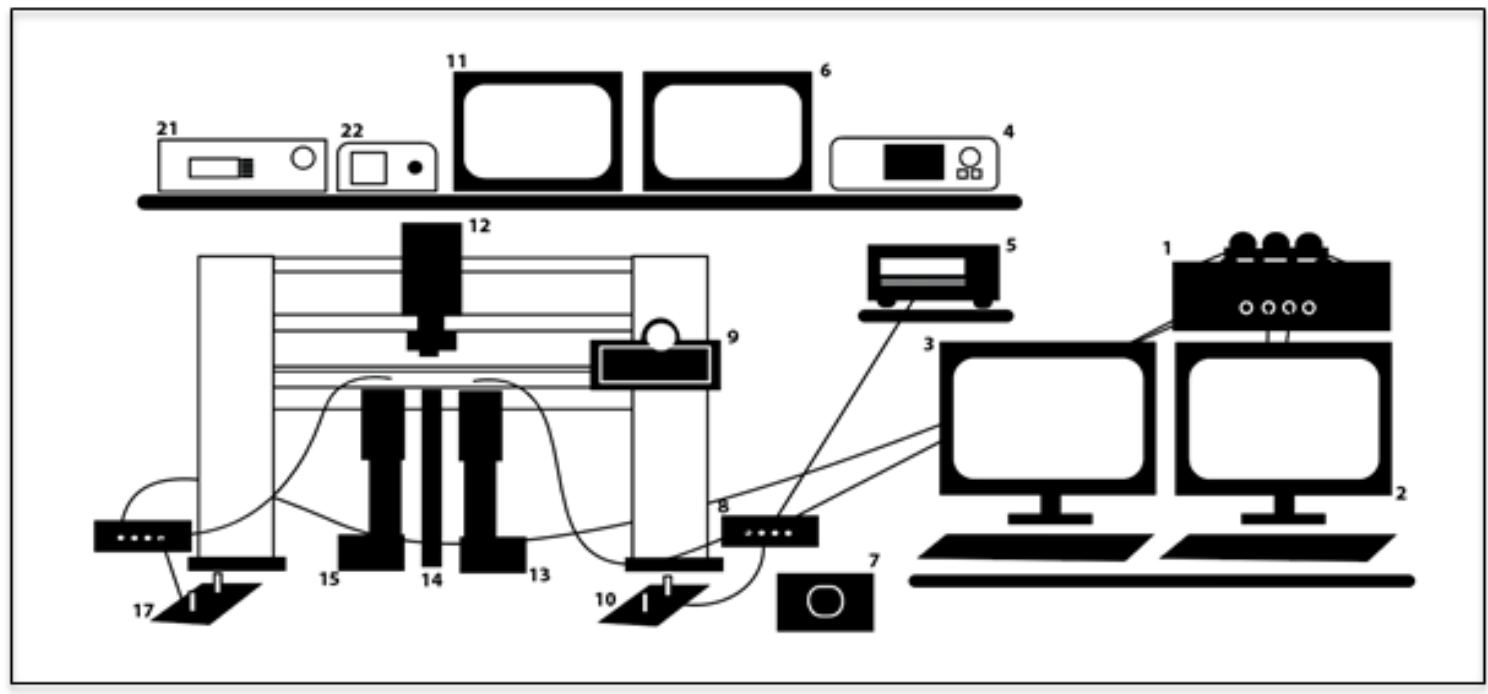

1. LUNA OVA 5000 Optical Vector Analyzer

2. Computer connected to LUNA

3. Computer controlling stage XYZ movement

4. EDFA (Erbium Doped Fiber Amplifier)
5. Newport Power meter

6. Display for IR horizontal view camera

7. Red central power button

8. Wire terminal between vector analyzer, power meter, and right fiber
10,17: Input switches for wire terminals

11. Display for overhead camera

12. Overhead camera

13. Right stage for right fiber

9. IR Camera with horizontal view
14. Center stage for photonic chip

Figure 4.1. Schematic of the set-up showing the testing tools used at MIT during the measurements. 


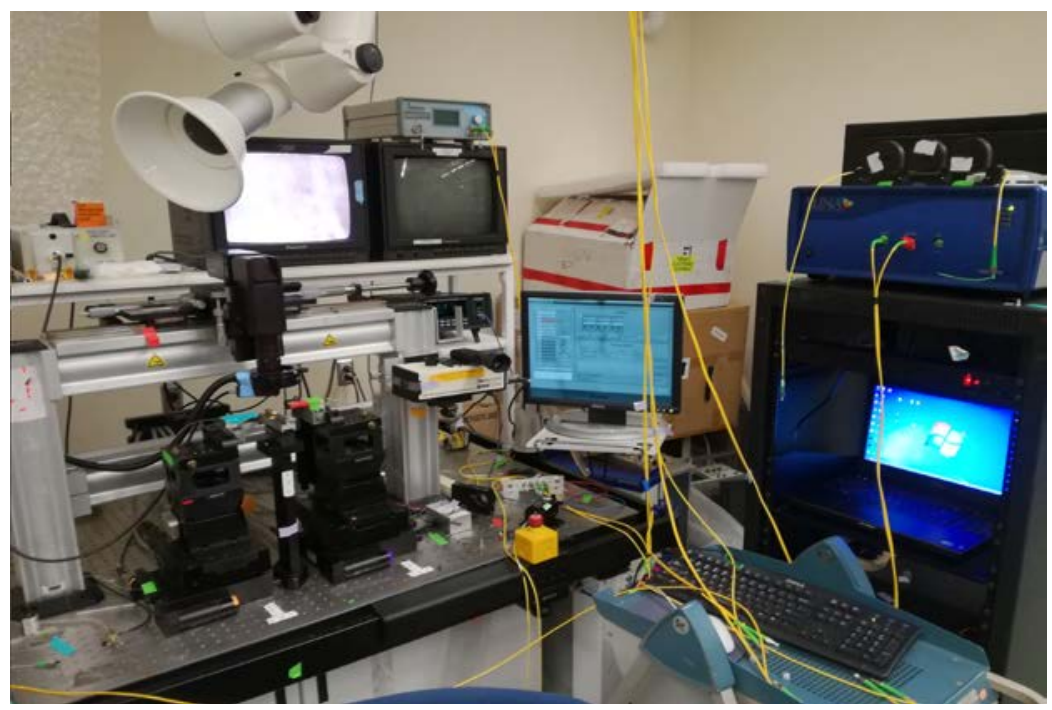

Figure 4.2. Photograph of the set-up showing the testing tools used at MIT during the measurements.

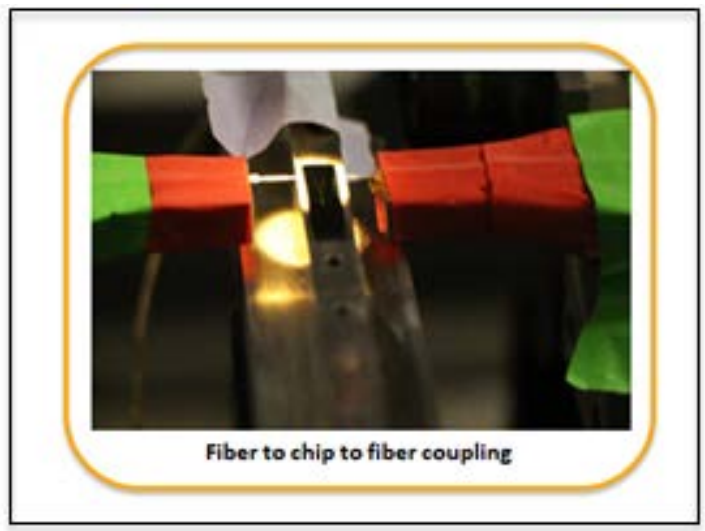

Figure 4.3. Photograph showing input and output fibers well-aligned to the chip.

The set of laboratory manuals created to teach the IP curriculum are widely usable documents for residential learning on MA and NY campuses, which are typically in four-year universities, community colleges and polytechnical institutions. In addition, they are available as a teaching tool to a larger global audience. This audience includes students around the world and veteran industry engineers seeking to be upskilled in TAP. The vision is that these laboratory manuals can be employed wherever integrated photonics is taught and where a need exists for photonics technician/engineer workforce training.

\subsection{Tools for PIC pedagogy}

In this section, a few important pedagogical tools that were used throughout the laboratory manuals are discussed. Included in each manual are: (a) specific text and visuals where necessary, (b) videos in the online version of the manual to better explain concepts, and to visually teach complicated procedural steps in the experiment, (c) a glossary section at the end for reference, because a new photonics vocabulary is introduced, and (d) a list of commonly occurring chip defects such as scratches, dust particles, cracks, and waveguide discontinuities, to help students to identify them right away, saving them valuable time when they are back in their workplaces.

\section{(a) Teaching through practice}

Instead of using a stand-alone lecture to teach PIC concepts, the idea is to reinforce learning by teaching students to perform hands-on testing in a laboratory setting, using the carefully crafted manuals as guides. An advantage is that as they learn concepts, technicians and engineers also become trained in testing. The laboratory manual is designed to teach 
a concept only when it is required for performing the test. For example, consider a simple circuit consisting of a laser, fiber, chip, and detector. PIC functionality cannot be understood without teaching about the confinement of light, typically a difficult concept for students to grasp. To teach confinement, the most basic building block is chosen - a straight waveguide with a high index core (silicon) and a lower index cladding (silicon-dioxide). An explanation for confinement is made using the wave approach. The next required building block in the circuit is a fiber, which is used to transport light from the laser source to the integrated waveguide. At this juncture, the concept of confinement of light is reinforced using the fiber as an example, but this time using the ray approach of total internal reflection. The next step is edge-coupling of a fiber to a straight waveguide, at which point several important concepts are taught. These include: (i) loss of light through reflection at the facet, (ii) size mismatch between fiber and waveguide, (iii) guided modes, and (iv) mode mismatch.

\section{(b) Structure of the laboratory manual}

The text in the manuals leads you through a careful step-by-step process in the characterization of each of the five fundamental building blocks. Every laboratory manual starts with a set of preliminary questions which the student can answer (10 minutes) based on the lecture-in-the-lab (30 minutes), prior to starting hands-on lab work (2 hours). For example, as a case-study, the laboratory manual that teaches about measurement of waveguide losses is considered. This manual uses spirals as the PIC device. In addition to the lecture-in-lab sections of the laboratory manual and the step-bystep explanations of the experiments, both pre-lab and post-lab exercises are added to encourage the student to recognize common themes and concepts throughout the various manuals. The exercises serve as both teaching and assessment tools. These features of the manuals, in addition to the hands-on experience, lead to a deeper, more intuitive understanding of IP, resulting in confident technicians and engineers, who become independent thinkers that are ready to effectively meet practical challenges, propose solutions to improve testing, and face automation.

\section{(c) Non-traditional methods adopted for teaching photonics}

To ensure that the laboratory manuals were lucid, they were created using a unique model (Fig. 5) as follows:

1. Technician students and engineering students worked together to build the manual

2. A postdoctoral associate who is:

- An expert in photonics, trained a team consisting of a technician, two undergraduate engineers, and a scientist, all from non-photonics fields. [The team was trained in PIC characterization in the laboratory, and was encouraged to ask questions during the training. These questions were then recorded and duly answered in the laboratory manuals, with added schematics and exercises that would enhance understanding]

- An OSA ambassador planning to pursue a career in academia

Salient features of this method are that:

1. It led to an effective parallel two-track curriculum for technicians and engineers

2. A PIC chip specifically tailored for education (deliberate introduction of faulty designs in the chip forces students to problem-solve), was used in the experiments.

Feedback from this process has led to the development of well-balanced teaching approaches serving a population with diverse backgrounds. Vocabulary unique to the field of photonics has been compiled into a glossary. 


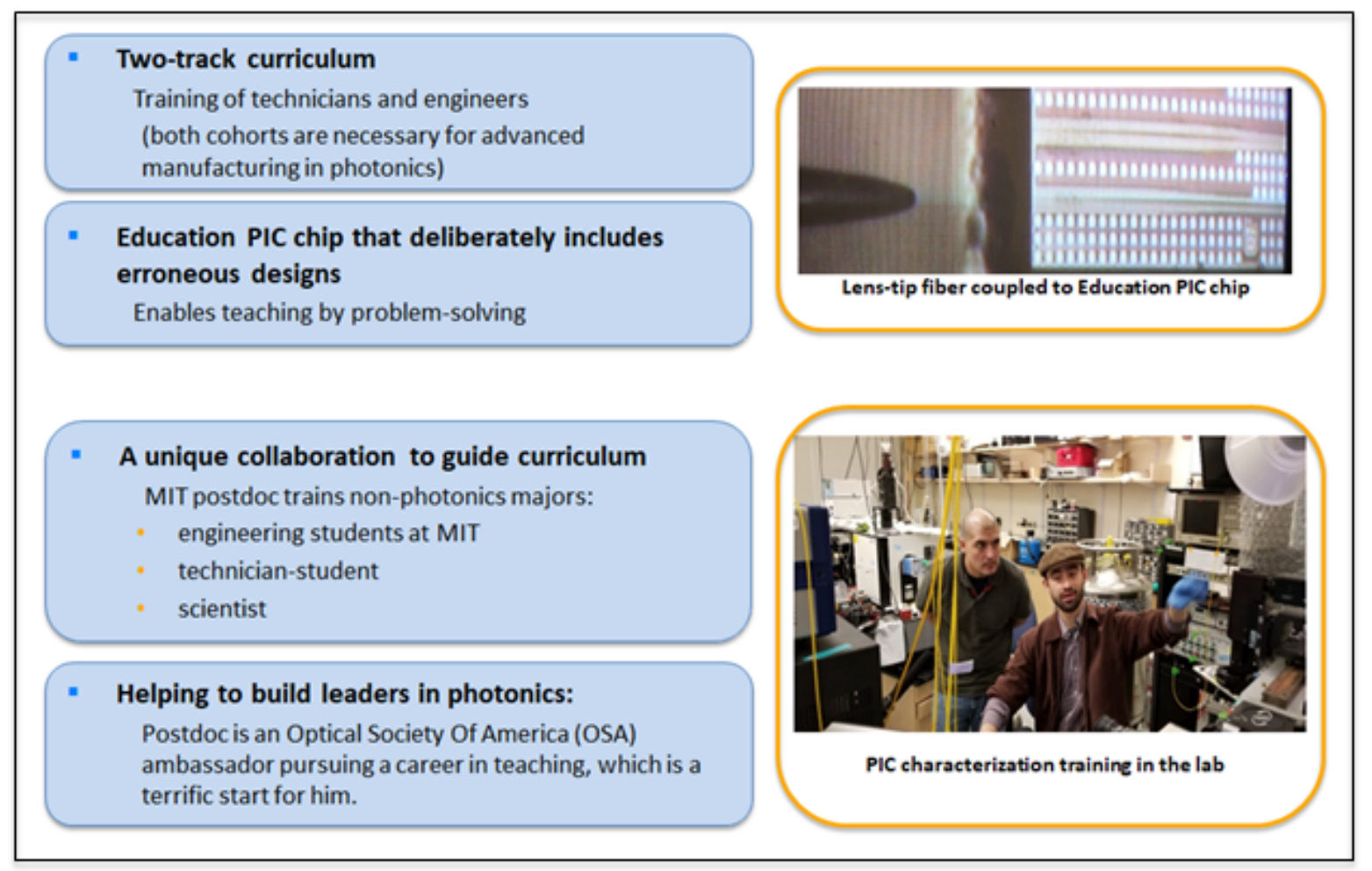

Figure 5. Non-traditional methods adopted for teaching photonics.

\section{(d) Drawing on existing approaches}

An existing model that was used to guide curriculum building and laboratory manual creation was an integrated photonics manual published and distributed by OP-TEC in 2016 (https://www.optecstore.org/products/integrated-photonicsevaluation-copy/). Features adapted from the OP-TEC manual include open-ended exercises to gauge reader understanding and detailed contextual information to help learning retention. While carrying over these useful features, the current manuals provide a variation in exercises. These were targeted to the specific experiment, and also categorized as pre- and post-lab exercises, to better assess (a) new learning, (b) recall, and (c) retention. The depth, accuracy, and clarity of the context provided has been enhanced by including PIC application examples. Also, interactive exercises such as data analysis using Python are included to enable the teaching of this very important aspect of photonics for technicians and engineers. Students are thus given tools to apply learned concepts to problem-solving in their respective fields. Post-lab exercises are introduced to reinforce learning. Answers will be provided to teachers for instruction and statistical information.

The laboratory manuals are generously populated with explanatory schematics, photographs and brief videos that show accurate depictions of the lab environment, demonstrate experimental procedures, and teach about device functionality. Additionally, TED-Ed videos, edX course modules and Virtual Lab simulations can be used to enhance teaching. In conjunction with the team creating laboratory manuals, a separate team at AIM Photonics Academy at MIT is developing a Virtual Lab, which includes game-based learning of PIC device functionality. An example of a waveguide with a $2 \mu \mathrm{m}$ bending radius is shown in Fig. 6. This simulation can also be made available to students, as a pre-lab exercise opportunity. 


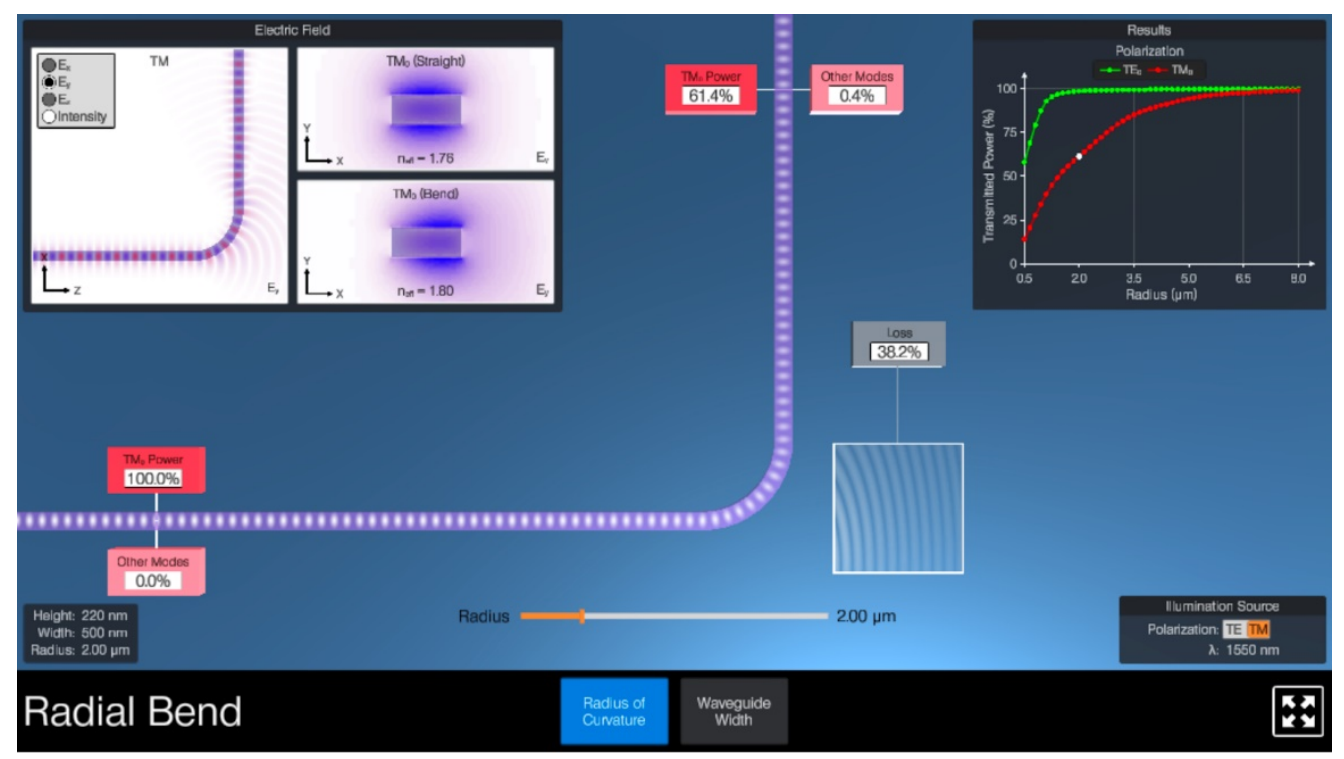

Figure 6. Virtual Lab as an auxiliary tool for the hands-on curriculum. Example of a bending radius of $2 \mu \mathrm{m}$.

\section{REAL LAB: HANDS-ON TRAINING AND DYNAMIC DOCUMENTION}

Several unique photonic testing skills that are important for a student to acquire are worth mentioning. We discuss seven of them here. For example, how to handle a chip, how to use precise mechanical equipment, and how to understand the mode displayed and the expected response of different components. Hands-on activities are designed to inspire confidence in a student's ability to handle equipment as well as acquire and interpret data. As a result, upon completion of the curriculum, a student will be better equipped to apply the skills and concepts they have learned.

\section{(i) Handling and placement of the chip}

Staging is a fundamental part of any chip measuring procedure. Proper set-up and alignment of components must occur to acquire accurate data. This critical skill is essential and, when mastered, increases the student's confidence. Hands-on capability is a unique feature and can be learned only by practice because no online training or book can sufficiently communicate the sense of orders of magnitude in mechanical displacement or power transmission measurements.

Handling photonic components requires familiarity and awareness of their function. For instance, when picking up a delicately fabricated integrated photonic chip designed for edge-coupling, there is a high risk of damaging the waveguide facets along the edges where the waveguides end, and this can lead to an increase in coupling losses. Efficient edgecoupling of light from a laser into the waveguide relies on the integrity of these delicate edge facets. Fig. 7 teaches important handling procedures, from which a novice technician/engineer can benefit. Following a specific procedure ensures the least possible damage to the waveguide facet when handling with tweezers. 


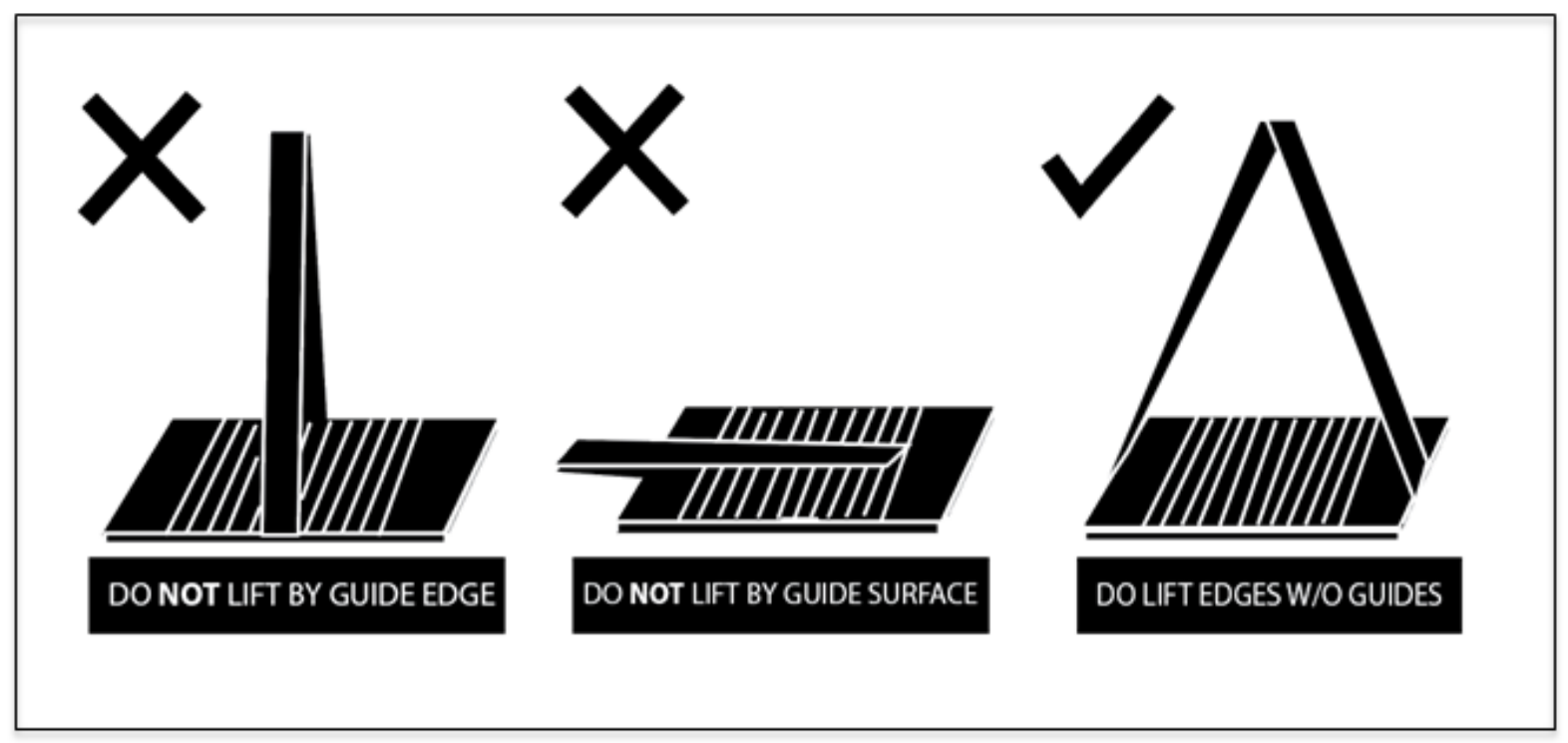

Figure 7. Correct handling of integrated photonics chip with tweezers. The integrity of the waveguide facets along the edges is crucial for accurate measurement. Thus, when lifting the chips, tweezers should not come in contact with waveguide facets.

While tweezers are designed for chip handling, it is still crucial for users to avoid lifting a chip in the wrong orientation. For a first time user, the chip may appear as a uniform piece since the facets are too small to spot. By having the user observe what appear to be parallel lines across the top of the chip indicates to them that the facets are at the ends of those lines. Placing the tweezers on the perpendicular sides from where the facets are located allows the user to know where to apply enough pressure to pick up the chip.

Once the chip is picked up, placing the chip on the staging area should be done in a single motion and over the shortest distance possible. This is achieved by simply having the sample holder as close to the stage as possible before picking it up. Once the chip is secure within the tweezers, it is carefully transferred to the staging area with the facet ends facing the input and output fibers on each side.

By placing the chip in this orientation, it sets up the alignment process; if the chip edges with facets on them are parallel to the edges of the stage, this will reduce the time it takes to optimize alignment later on in the process, in particular, when moving from one waveguide under test to the adjacent one.

While the practice of placing a chip on a stage can appear to be a simple task, there are many precautions that should be taken and proper protocols must be considered to protect the integrity of the equipment. These details are emphasized in the laboratory manuals to create mindfulness for the safety of the user, the equipment, and the samples.

\section{(ii) Bringing the fiber into focus when near chip}

The concept of waveguiding has been taught traditionally using fiber optics as an example, and corresponds to low index contrast structures $(\Delta \mathrm{n}=0.005)$ that have relatively large cross-sections, for instance, single mode fibers (SMF) which have cores on the order of $10 \mu \mathrm{m}$ diameter (Fig. 8). One of the main consequences of using a silicon waveguide and its native oxide as cladding is the large refractive index contrast $(\Delta \mathrm{n}=2)$ that corresponds to single mode structures on a the submicron scale. The absence of efficient and low cost integrated on-chip lasers makes the complicated coupling from an external source to the silicon chip a necessity. This is typically done with either free space optics (microscope objective) or fibers (lensed or cleaved). In the laboratory manuals, a lensed fiber is used to inject a laser beam into a chip via edge coupling (Fig. 8). 

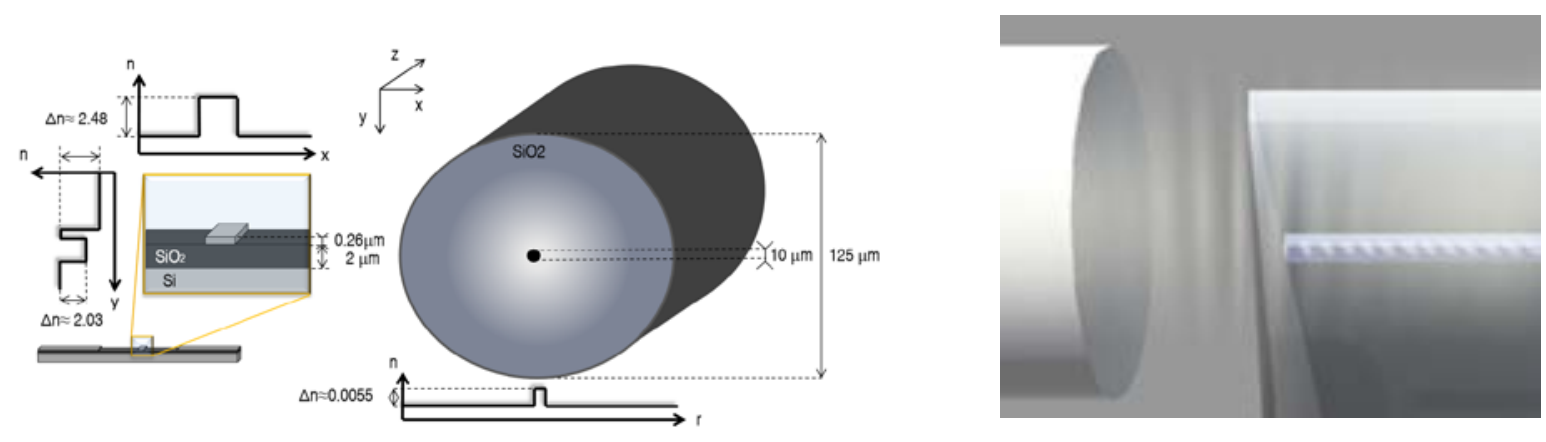

Figure 8. Scaled comparison between the size and refractive index profiles of a single mode fiber and a single mode SOI waveguide [6].

This coupling is achieved manually by visual inspection. Specifically, by bringing the chip into the focal plane of the microscope, using an overhead camera (Fig. 9), so that each waveguide is visible. Maneuvering the fiber to the "start position command" in the position editor via the mechanical staging system, is controlled by Ficontec software. Teaching the process of approaching and aligning the input and output fibers is fundamental to protect the sample and the lensed fiber.

What creates difficulty is that the fiber is about the width of a human hair and is hard to focus under the camera's objective alone. Creating this process allows room for errors and helps the user to scale the distance covered when moving only a few microns at a time. Learning how and when to move the fiber also sharpens skills in component use. Students are asked to note that depending on the fabrication of the chip there may be a burr visible just under the edge with the facets which can be seen on the microscope display. This burr can create visibility issues due to low contrast between the burr and the fiber tip.

The concept of a taper on a chip is introduced at this point and a few open ended exercises are presented to the student, asking about the function of a taper function. Learning by doing is emphasized when students compare the power transmitted using a taper to not using one, and find the former being higher. At the edge of the chip, the taper is approximately $20 \mu \mathrm{m}$ wide. This causes the user to lose sight of the fiber, becoming an important exercise for the student to improve illumination conditions, i.e. using a microscope lamp to minimize the problem.

\section{(iii) Moving or “walking” the fiber towards the chip}

Aligning the fiber to the chip is a process that requires intuition and care. As seen in the previous subsection and Fig. 8, the fiber tip is relatively large compared to the cross-section of the waveguide. Additionally, the chips are fragile and expensive. Explicit steps are included in the laboratory manuals, teaching the student about the importance of following a sequence of movements to safely align a fiber to the chip without damaging any equipment. Even though systems that automatically align the fiber to the chip are commercially available today, it is important that the student gets familiar with the orders of magnitude regarding the displacements allowed and the limits for the procedures, so as not to damage any electrical, optical or mechanical components. At the end of this training, both technicians and engineers are expected to have developed confidence and intuition on approaching the chip with a fiber, and should be able to set up for chip measurements efficiently, in both manual and automatic modes. This particular crucial step requires practical exercises. Hands-on skills are assessed when the alignment process is demonstrated by a student to the teacher.

Once the fiber is in focus with respect to the chip, the next step to teach the student is to allow the fiber to slowly and carefully approach the chip facet. Typically, a $300 \mu \mathrm{m}$ distance is used to allow the student to move at a safe pace as the fiber approaches a chip that is not yet visible on the screen. The distance is adjusted once the fiber and the chip are both visible in the same shot, then the displacement distance is gradually decreased. On the test set-up at MIT, once the fiber has approached the chip, a minimum distance is set in the jog panel equal to $0.5 \mathrm{um}$ (Fig. 9). Such hands-on steps are specific to the tools used in each laboratory. No matter the set of tools used, alignment is the most critical step and hence is taught one-on-one to the student; assessment of this skill is also performed one-on-one. Schematics in the manual can reinforce this training. Technicians and engineers need to continue to practice these skills to make it second nature. 


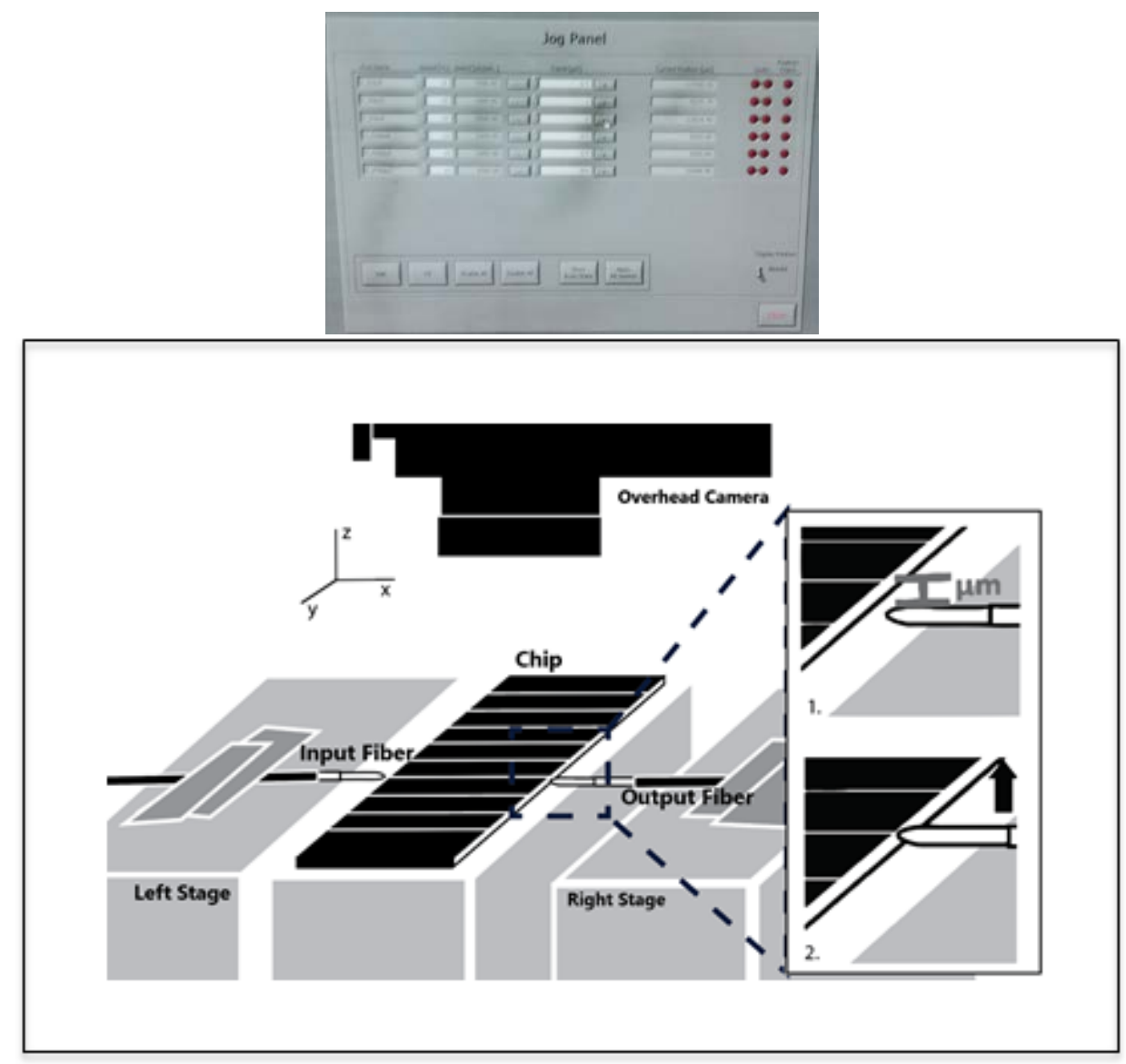

Figure 9. Jog Panel control of the mechanical stage and butt-coupled fiber/stage set-up. Note that a stripped fiber with an exposed core is edge-coupled to a waveguide. 1 . The output fiber tip is located several microns directly below the target waveguide. 2 . The output fiber tip is translated up along the z-axis.

These steps are used to assist new or inexperienced users to maneuver the input and output fibers with little to no incidents of fiber, chip or tool damage. The AIM education chip edge also presents a challenge. Due to the manufacturing process of the chip, on its facet edge, there is a 15 to 20 um ledge extending outward, below the facets. In the monitor, it is observed as a dark region that provides little to no contrast once the fiber tip is within that region (Fig. 10). Understanding how much to move the fiber by approaching the facet at such a close distance is key to not damaging the components and obtaining good measurements. For engineers, experiencing first-hand the difficulty in obtaining sufficient contrast between the fiber and the ledge below will lead to a lesson that design for measurement must strictly be followed.

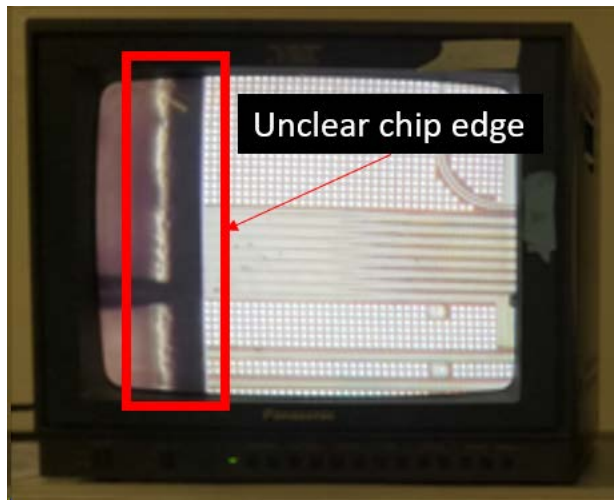

Figure 10. Image of a fiber that has been aligned with a waveguide on a chip. 


\section{(iv) Teaching about a confined optical mode and how to find it}

An optical mode is a physical description of the transmission of energy/information in a waveguide. The modes are solutions for the propagation of light and their shapes are found by solving the Maxwell equations (eigenvalue problem). Fig. 11 depicts the different components of the fundamental TE electromagnetic mode (electric and magnetic fields) in a SOI strip (fully etched) waveguide. The silicon thickness in the AIM education chip is $220 \mathrm{~nm}$ and the width of the waveguide is $400 \mathrm{~nm}$. It is noteworthy that $E_{x}$ is the dominant component. Interaction with the waveguide sidewalls highlights the importance of proper fabrication quality.

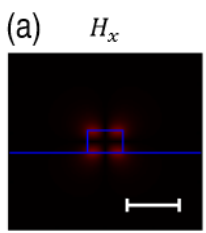

$E_{x}$

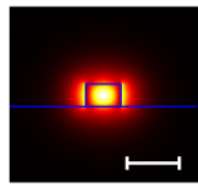

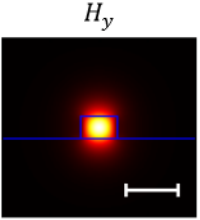

$E_{y}$

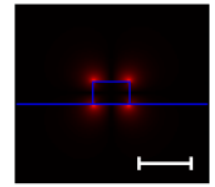

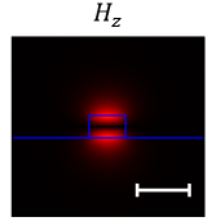

$E_{z}$

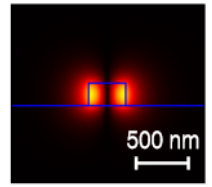

Figure 11. Magnetic and electric field components of the fundamental TE mode in a SOI strip waveguide.

Before performing transmission measurements, understanding that light being transmitted through the waveguide is not all the same, is important. In the laboratory, light transmitted in the upper cladding, or too far into the substrate below the under cladding, will transmit different modes, which can be physically observed by the operator. The single mode fiber injects a single mode of light onto the chip's facet. When this ray is incident exactly on the chip's facet, it will cause a certain mode to be observed at the output through an infrared camera. A mode is a path that a light ray takes through a waveguide. If the path from fiber to chip strikes above the waveguide, the image viewed on the infrared camera displays a missing bottom section. If the input fiber is aligned too low, the mode is incident on the substrate and the camera shows an image with a top section missing. The user should expect to observe a solid circle, which is achieved by optimizing the input fiber alignment to the waveguide facet using the jog panel found on the staging system software. The optimization of this alignment also reduces the number of fringes which are observed in real time. These fringes are due to interference of light within the waveguide. This allows the student to obtain a mode without any interference, that will be incident on the system detector.

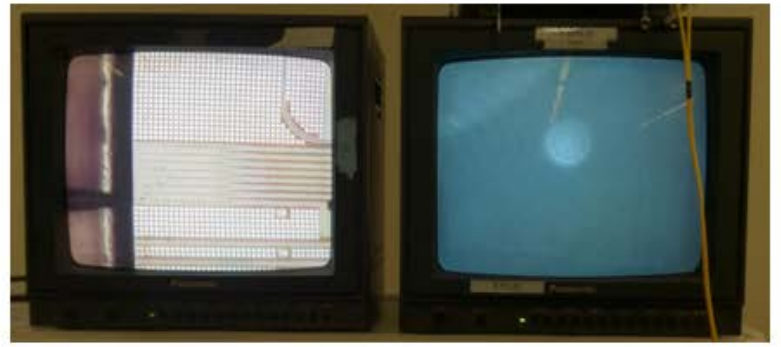

Good Mode

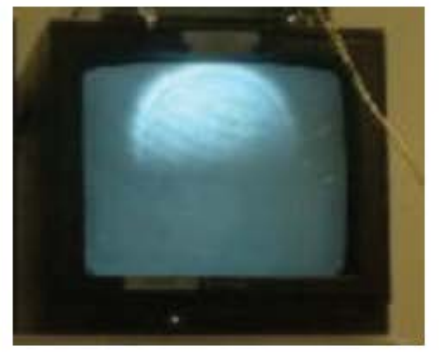

Waveguide Low

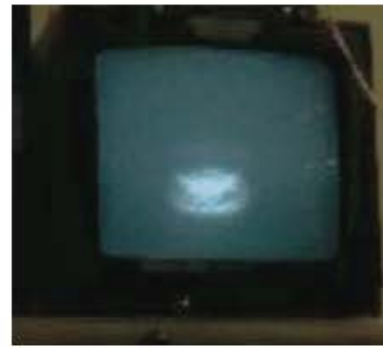

Waveguide High

Figure 12. Displays during calibration and their associated modes. Left display is a top view of the fiber being aligned with the chip. The different images captured with the infrared camera are presented when the fiber is: (i) at the right height (Good mode), (ii) above the waveguide (Waveguide low) or below the waveguide (Waveguide high).

A lot of how to achieve this mode depends on the familiarity the user has with the equipment and a tacit skill in maneuvering the optical fiber a few microns at a time. A certain feel for the machine is also developed over time and with practice. Demonstration of the three stages of alignment will be used to assess a student's skills. 


\section{(v) Spirals to measure the propagation losses}

Spiral waveguides have been widely used in integrated photonics particularly for sensing applications as the propagation length is increased while keeping the circuit compact. Teaching how to test such a device is important in real applications. In Fig. 13, an example of the layout for spirals is presented.

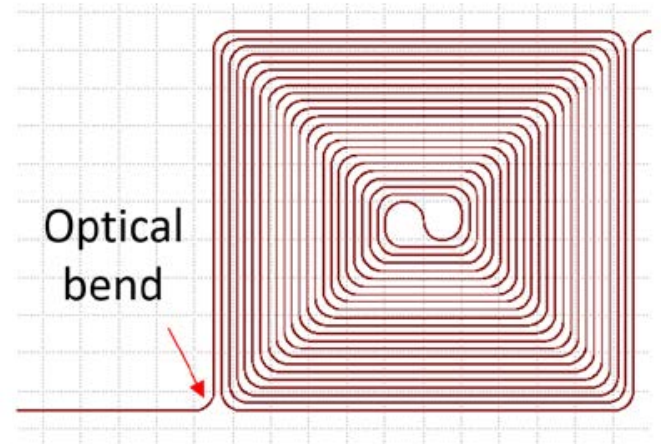

Figure 13. Layout of a spiral device of $\sim 200$ X $200 \mu \mathrm{m}^{2}$ and $\sim 1 \mathrm{~cm}$ propagation length.

Properly displaying the optical transmission is fundamental to understanding the wavelength dependence of the waveguide and also for post-processing steps to calculate the propagation losses via the "cut-back" (variable length) method. Different software was used to analyze the data, nevertheless, free software will be leveraged, so the student will have access to a set of Python scripts that will allow remote post-treatment. In Fig. 14, the transmission for different waveguide lengths is presented. Also, an interpolation of the data is displayed.
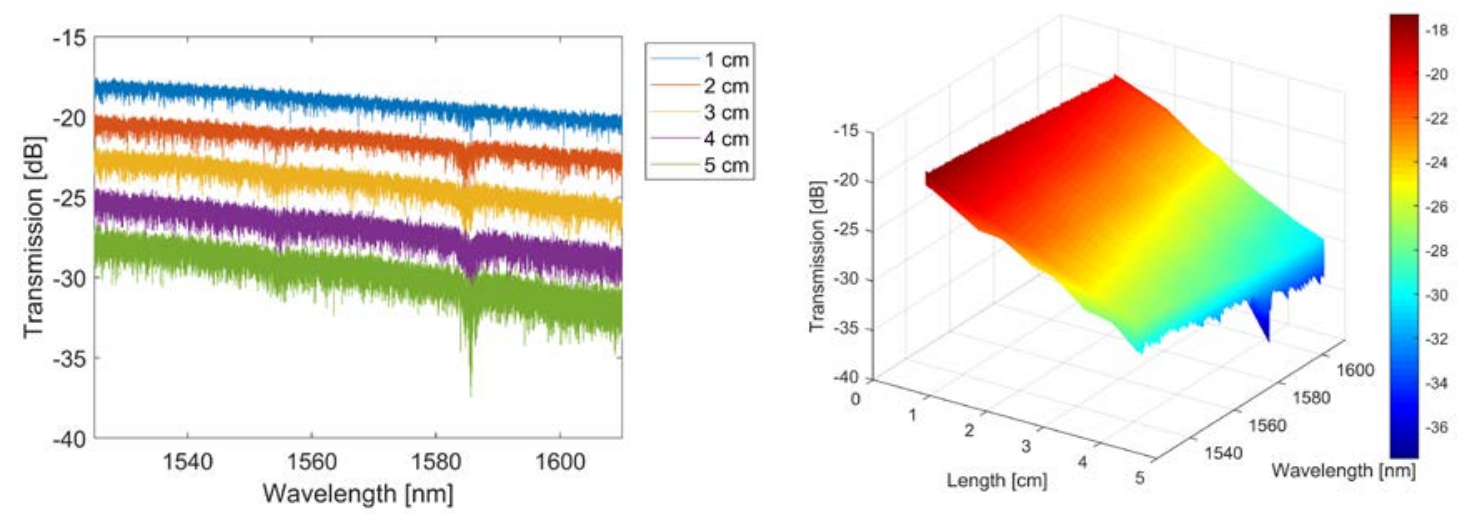

Figure 14. Left: Optical transmission as a function of wavelength for different propagation lengths from spirals introduced in previous section. Right: 3D transmission plot extrapolation.

With the free Python codes offered, students can visualize important trends and begin to understand why these trends may arise. For example, from Fig. 14, a student may notice the dips in transmission around $1585 \mathrm{~nm}$, which could be interpreted as a broad absorption peak within the waveguides, increasing with waveguide length. In the post-lab exercises, the lab manual will have questions about the pattern. Also, it will be possible for the student to fix a wavelength and find the propagation losses by a linear regression as shown in Fig. 15 for a $1560 \mathrm{~nm}$ wavelength. 


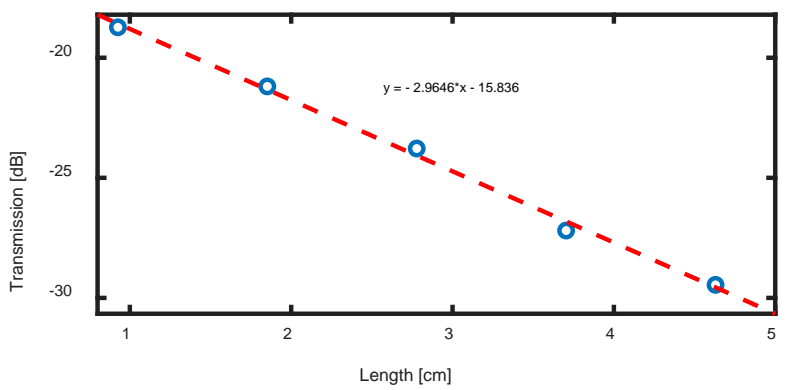

Figure 15. Linear regression at $1560 \mathrm{~nm}$, giving propagation losses of $2.96 \mathrm{~dB} / \mathrm{cm}$ in SOI waveguides. The coupling losses are found to be $\sim 16 \mathrm{~dB}$ in total.

\section{(vi) Ring resonators}

As another example of rich key building blocks in integrated photonics, ring resonators (RRs) correspond to fundamental geometries used in all integrated applications, from sensing to quantum photon pair generation [7]. However, grasping the fundamentals of RR device functionality is difficult for a student. Hence, blended learning (a classroom lecture, a virtual lab simulation or an edX video), is advised to introduce the required theoretical background explaining the fundamental concepts behind these structures. Two fundamental parameters are varied to understand concepts such as free spectral range (FSR), quality factor (Q), over-coupling, critical coupling, and under-coupling. In Fig. 16, two essential parameters are reinforced, namely ring radius and coupling gaps.

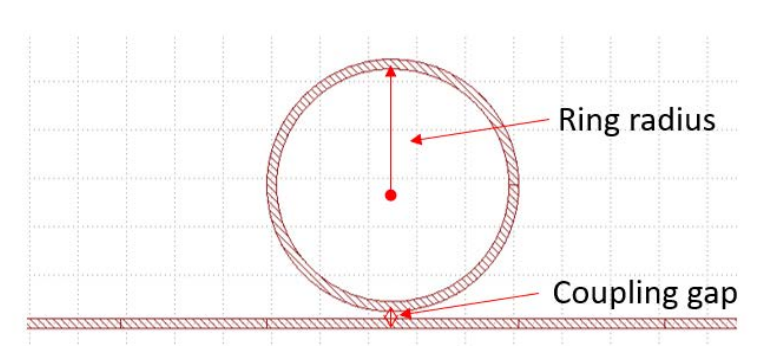

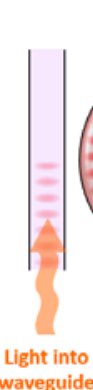

waveguide
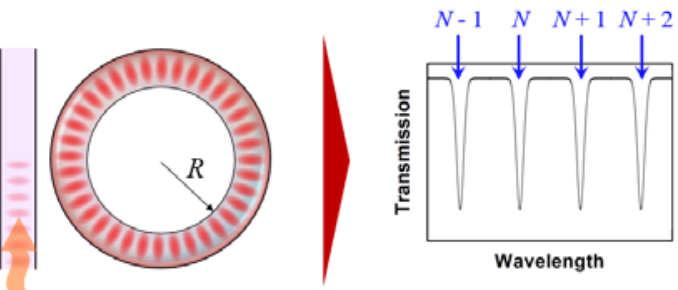

Resonant condition:

$N \cdot \lambda=n_{\mathrm{eff}} \cdot 2 \pi R=n_{\mathrm{eff}} L \quad N=1,2,3 \ldots$

Figure 16. Left: Diagram of a ring resonator highlighting its radius and gap. Right: Behavior of an ideal ring resonator. (Images courtesy Samuel Serna and JJ Hu)

Similar to the previous structures, ring resonators have a set of Python software routines available to analyze the data. As an example, experimental results from a silicon on insulator chip with a ring resonator with a radius of 5 micron and a coupling gap of $300 \mathrm{~nm}$ is presented in Fig. 17. 

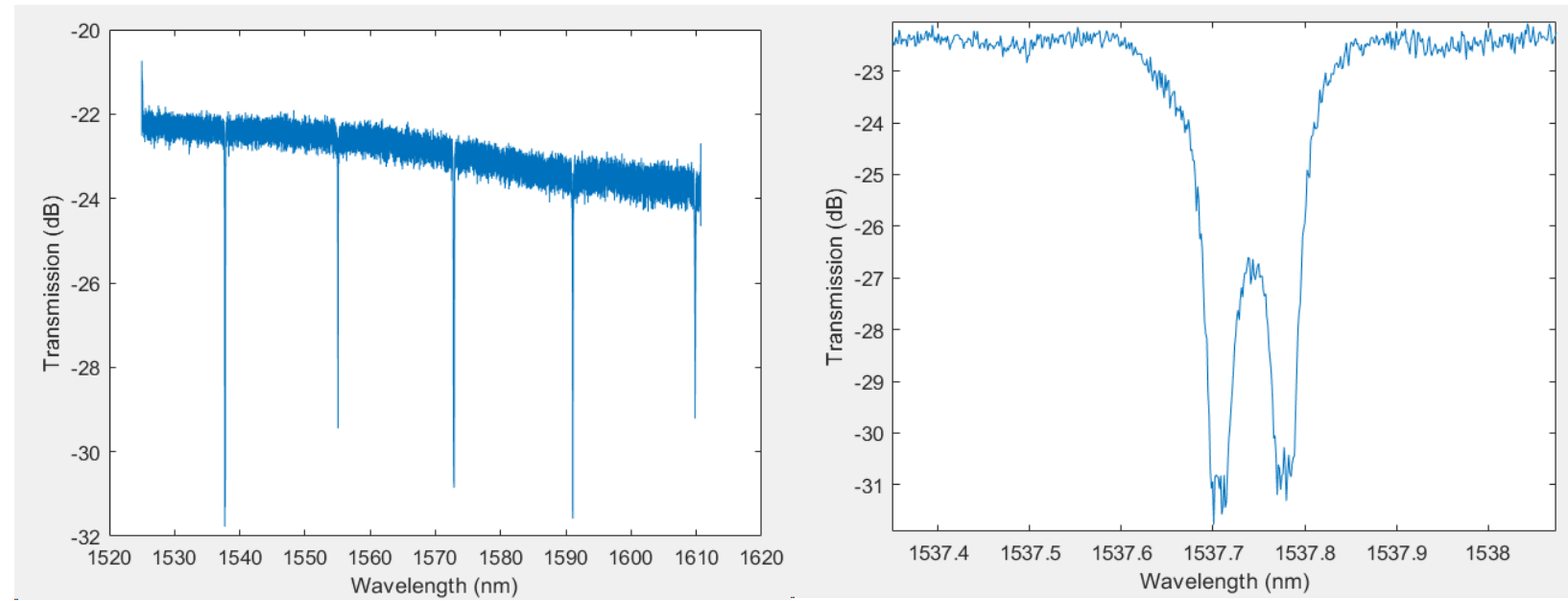

Figure 17. Experimental broadband transmission from a ring resonator and a zoom-in on one specific resonance.

After the experiments, students will likely see the presence of two dips, as shown in the zoomed-in version of the resonance peak on the RHS of Fig. 17. Post-lab exercises are used to engage students regarding the origin of these two dips as opposed to a single resonance peak. Students can be taught about limits in fabrication processing which lead to the observed anomaly. Non-uniformity of edge roughness and fabrication features, leads to counter-propagating waves in RRs. A quantitative explanation can be introduced to engineering students by the use of matricial representation of the resonator.

For the ring resonators, a Lorentzian fit is applied to the various peaks, and the best Lorentzian function is shared with the student. From this function, a student can learn that the resonant frequencies and length of the waveguide, amongst other factors, all affect the transmission loss. Likewise, the linear regression shown above would highlight how the length of a waveguide and the transmission loss are related and can also be used to find the propagation losses of a waveguide.

\section{(vii) Waveguides through a boot camp training session}

As an intermediate goal, the laboratory manuals will be used in a boot camp, after which students can expect to leave with an understanding of how and why waveguides are used to transmit information and the current limitations and challenges of using this technology. Students should also gain practical, hands-on experience on how to use different equipment relevant to the technology. Finally, an understanding of how to optimize the power transmission of a waveguide for different applications should be understood.

\section{STUDENT ASSESSMENT}

Included in each laboratory manual are pre-lab exercises that aim to draw the student into the topic, and post-lab exercises that are used as assessment tools to better understand learning outcomes such as new learning, recall, and retention [8]. A few of these exercises can encourage creativity and out-of-the-box thinking as well. Listed below are seven ways in which we hope to evaluate student learning through these laboratory experiments.

\section{(a) Promoting lateral out-of-the-box thinking}

An example of a targeted exercise that can generate out-of-the-box thinking is discussed here. In the laboratory manual that teaches about the measurement of transmission losses using spirals on a chip, an exercise encourages students to question the use of curvy spirals as opposed to the straight waveguides they encountered in a previous laboratory experiment. A simple question about the reason for using curves in photonic circuits can trigger a student's thought process in a direction conducive to questioning several related nuances. For example, some follow-up questions raised by the students may be: (a) How are curves designed and fabricated?; (b) How do photons propagate at waveguide bends compared to electron flow within curved wires?; (c) Are there losses associated with bending?; and (d) Is it suitable for specific applications since bends allow a smaller footprint? This thinking is encouraged by having the student record not only their answer, but also additional questions raised. Assessment is based on the extent of lateral thought. 


\section{(b) Evaluation of deductive reasoning}

The ability to perform deductive reasoning is an important skill in technicians and engineers who are required to problemsolve daily. A metric for evaluating a student's grasp of the material can be their deductive reasoning ability. Some exercises have been tailored to perform this evaluation. For example, when measuring the power transmitted through a waveguide, the student learns about coupling losses at facets, scattering losses due to edge roughness, and absorption losses within a material. An exercise asking about what they might expect to see when the length of the waveguide is increased should lead, by deductive reasoning, to a conclusion that losses will increase with length. A technician student is expected to identify which of these losses will increase and how, based on previously presented material about etch processing conditions and materials comprising the waveguide system. An expected answer from such a student is that as length increases, losses must increase because of absorption losses within the entire waveguide length and scattering losses along the edges. An astute engineering student, on the other hand, will be expected to deduce that a plot of transmission versus length will yield quantitative information about insertion (total) loss and coupling loss.

\section{(c) Assessment of the application of prior comprehension}

Application of prior comprehension indicates a student's upward trajectory in learning. An exercise to evaluate the application of prior comprehension is demonstrated by using confinement of light as an example. Since light confinement is taught in the first laboratory manual, while transmission loss is taught in a future lab, an exercise which asks whether the student expects to see a difference in transmission between a symmetrically-clad waveguide and an asymmetricallyclad waveguide is appropriate to evaluate application ability. Both technician and engineer students are expected to answer that the symmetrically clad waveguides are less 'lossy' since the mode is more confined within the low-loss waveguide. When the cladding is asymmetric, the mode gets pulled to the higher index cladding side and interacts with the interface roughness.

\section{(d) Evaluation of blended learning outcome}

If the student had previously learned to use a virtual lab simulation in which waveguide width was a variable parameter, this implies that the student was presented an intuition regarding confinement of light. To assess how much of the online learning of light confinement within a waveguide could be applied to a hands-on learning environment, an exercise in the manual asks if a student expects the transmission loss to be affected whether light remained outside the waveguide for smaller waveguide widths versus inside the waveguide for larger waveguide widths. Both technicians and engineers are expected to apply the intuition gained in a virtual lab setting to answer that confinement within the waveguide would be greater for wider waveguide widths, minimizing interaction with sidewall roughness, leading to lower loss.

\section{(e) Evaluation of the ability to use tools in a photonics testing laboratory}

Since the laboratory manuals teach testing procedures, operating skills that technicians and engineers must learn are very important to assess, and are done using exercises introduced throughout the lab manuals. These exercises include questions about alignment procedures using automated and manual alignment stages, fiber use and maintenance, sample mounting skills, and laser and photodetector use.

\section{(f) Assessment of data analysis skills}

Based on the industry skills-needs survey, both engineers and technicians are required to analyze data collected in a photonics testing laboratory and present it to their team. Python-based software programs are taught and different sets of data are analyzed. Exercises determine whether or not a student was able to interpret the data meaningfully and accurately. An engineering student may be asked to identify and explain observed measurement anomalies. Since known faulty designs have been incorporated in the education chip, an assessment is possible based on information provided in the lesson plans for teachers.

\section{(g) Grading and certification}

Students are graded based on their:

- $\quad$ Demonstration of tool use to characterize PICs

- $\quad$ Analysis and interpretation of data obtained for each PIC

- $\quad$ Ability to answer questions (exercises at the end of each lab, with extra-credit challenges) 
Upon successful completion of the training, a student obtains a certificate from one of the following places: LEAP center, community college, 4-year college, research institution or company - wherever a student has been trained. Since IEEE certification is highly desirable because it can be standardized, an attempt will be made to accomplish this through a collaboration with community colleges.

\section{CURRICULUM ADOPTION}

Either the entire curriculum or parts of it can serve in a pre-existing campus course to introduce students to PIC characterization, or can be used to conduct a photonics bootcamp. At MIT, we have planned to use it in both ways. A standard microelectronics processing course will be used in fall 2019, to introduce students to photonics packaging and testing. Also, a pilot photonics boot-camp will be held at MIT in January 2020, for MIT students and for other members of the greater photonics fraternity. Lessons learned from using this curriculum as a teaching tool will be used to improve it. Lab courses and/or boot camps will also be offered elsewhere by the MA and NY colleges on our team. Global instructors may or may not have experience in the field, and so the curriculum will include guidance for instructors. Video and audio files can also be shared by instructors.

If this training were placed into a different setting, say a semester-long photonics course, these laboratory experiments may be done bi-weekly, and teaching can proceed in a modular fashion. Experiments can be supplemented by lectures on the electromagnetic concepts that underlie the technology, on the materials science and fabrication techniques, and on the hardware and software systems that interface with these photonic circuits in current applications. In this type of setting, one could spend more time on the entire lifetime of a device: from the design, to the fabrication, to functionality testing, to assembly and packaging. Also taught is a statistical study of variations in the data obtained by different users, under different environmental conditions and with sample-aging, among others. Students could spend more time on the theory in the beginning, then fabricate/design their own chips, and finally test them using methods contained in the manuals.

\section{CONCLUSIONS}

A modular laboratory curriculum is created that can be disseminated around the world to teach fundamental photonics concepts. The goal is to train technicians and/or engineers in the field of integrated photonics, in order to enable them to be effective in their workplaces. The curriculum comprising five laboratory manuals can be implemented either as (i) a stand-alone laboratory course, or (ii) in a blended course that incorporates online or live lectures, demonstrations and visualizations, combined with a laboratory experience, to build a strong foundation of knowledge, comprehension and application. Pre- and post-lab exercises provide an intuitive grasp of the material, aid lateral thinking and are used to evaluate and assess student comprehension of fundamental concepts and student ability to analyze and interpret data.

\section{Acknowledgements}

We would like to acknowledge our team's strong commitment to photonics education and workforce development. Our complementary partnership was essential in building a curriculum that could be used to serve a diverse population. RIT led the design of the education chip in collaboration with BU and UR. MIT led the testing of the education chip and building the boot camp curriculum in collaboration with RIT and UR. As early adopters of the boot camp curriculum, WPI, QCC, Stonehill, and BSU offered feedback on how best to adjust for their own student populations. Direct feedback from diverse students, technicians, and independent consultants was invaluable. Our state government partners (NY and MA), along with our federal government partners (AIM Photonics Institute, DoD and OSD), have provided a vision for the future of integrated photonics, and have directed some resources to accomplish such a vision. We also acknowledge partial support of this project by Lockheed Martin, MIT, and an ONR-MEEP through grant number N00014-18-1-2890.

This material is based on research conducted under AIM Photonics and sponsored by Air Force Research Laboratory via agreement number FA8650-15-2-5220. The U.S. Government is authorized to reproduce and distribute reprints for Governmental purposes notwithstanding any copyright notation thereon. The views and conclusions contained herein are those of the authors and should not be interpreted as necessarily representing the official policies or endorsements, either expressed or implied, of Air Force Research Laboratory or the U.S. Government. 


\section{REFERENCES}

[1] Vivien, L., \& Pavesi, L. (Eds.). "Handbook of silicon photonics”. Taylor \& Francis. (2016).

[2] B.E.A. Saleh and M.C. Teich, "Fundamentals of Photonics”, John Wiley \& Sons, New York, (1991).

[3] Serna, S., and Dubreuil, N., "Bi-directional top-hat D-Scan: single beam accurate characterization of nonlinear waveguides”, Optics letters, 42(16), 3072-3075 (2017).

[4] Zhang, W., Serna, S., Le Roux, X., Alonso-Ramos, C., Vivien, L., and Cassan, E., "Analysis of silicon-on-insulator slot waveguide ring resonators targeting high Q-factors”, Optics letters, 40(23), 5566-5569 (2015).

[5] Marchetti, R., Lacava, C., Carroll, L., Gradkowski, K., and Minzioni, P., "Coupling strategies for silicon photonics integrated chips”, Photonics Research, 7(2), 201-239 (2019).

[6] Serna, S., "Design and characterization of Silicon Photonic structures for third order nonlinear effects", (Doctoral dissertation, Université Paris-Saclay) (2016).

[7] Bogaerts, W., De Heyn, P., Van Vaerenbergh, T., De Vos, K., Kumar Selvaraja, S., Claes, T., ... \& Baets, R., "Silicon microring resonators”, Laser \& Photonics Reviews, 6(1), 47-73 (2012).

[8] Bloom, B.S., Englehart, M.D., Furst, E.J., Hill, W.H. and Krathwohl, D.R., “Taxonomy of Educational Objectives: The Classification of Educational Objectives”, Handbook I: Cognitive Domain, David McKay, New York. (1956). 